\title{
Antimania-Like Effect of Panax ginseng Regulating the Glutamatergic Neurotransmission in REM-Sleep Deprivation Rats
}

\author{
Kang Hyun Leem, ${ }^{1}$ Sang A. Kim, ${ }^{2}$ and Hae Jeong Park $\mathbb{D}^{3}$ \\ ${ }^{1}$ College of Korean Medicine, Semyung University, Jecheon 27136, Republic of Korea \\ ${ }^{2}$ Department of Biomedical Science, Graduate School of Kyung Hee University, Seoul 02447, Republic of Korea \\ ${ }^{3}$ Department of Pharmacology, School of Medicine, Kyung Hee University, Seoul 02447, Republic of Korea
}

Correspondence should be addressed to Hae Jeong Park; hjpark17@khu.ac.kr

Received 1 May 2020; Revised 29 August 2020; Accepted 28 September 2020; Published 17 October 2020

Academic Editor: Mauro S. Oliveira

Copyright (c) 2020 Kang Hyun Leem et al. This is an open access article distributed under the Creative Commons Attribution License, which permits unrestricted use, distribution, and reproduction in any medium, provided the original work is properly cited.

\begin{abstract}
Previous studies have shown the therapeutic properties of ginseng and ginsenosides on hyperactive and impulsive behaviors in several psychiatric diseases. Herein, we investigated the effect of Panax ginseng Meyer (PG) on hyperactive/impulsive behaviors in a manic-like animal model, sleep deprivation (SD) rats. Male rats were sleep-deprived for $48 \mathrm{~h}$, and PG (200 mg/kg) was administered for 4 days, from 2 days prior to the start of SD to the end date of SD. The elevated plus maze (EPM) test showed that PG alleviated the increased frequency of entries into and spent time within open arms by SD. In order to investigate the molecular mechanism on this effect of PG, we assessed differentially expressed genes (DEGs) in the prefrontal cortex of PGtreated SD rats using RNA sequencing (RNA-seq) and performed gene-enrichment analysis for DEGs. The gene-enrichment analysis showed that PG most prominently affected the glutamatergic synapse pathway. Among the glutamatergic synapse pathway genes, particularly, PG enhanced the expressions of glutamate transporter Slcla3 and Slcla2 reduced in SD rats. Moreover, we found that PG could inhibit the SD-induced phosphorylation of the NR2A subunit of the NMDA receptor. These results suggested that PG might have a therapeutic effect against the manic-like behaviors, regulating the glutamatergic neurotransmission.
\end{abstract}

\section{Introduction}

Sleep disturbance is the most frequent symptom in manic episodes and a relapse factor of manic episodes in bipolar disorder (BPD) $[1,2]$. In rodents, sleep deprivation (SD) has been considered as an environmental mania model $[3,4]$. Indeed, in rats and mice, SD rodents have shown maniclike behaviors, including locomotor hyperactivity, hypersexuality, irritability, and aggressiveness, as observed during the manic phase in patients with BPD $[3,4]$. In addition, treatments of mood stabilizers such as lithium and valproic acid, which are have been used for relieving in the manic episodes in BPD, reduced these manic-like behaviors in SD animals [5-7]. Neurochemical alterations in SD animals were also represented, similar to patients during the manic phase in BPD. In the brains of SD animals, the dopaminergic hyperactivity, the increased activity of protein kinase $\mathrm{C}$, and the decreased levels of brain-derived neurotrophic factor (BDNF) were observed $[5,8-10]$.

Ginseng is a popular herbal medicine widely used for multiple pharmacological functions such as anticancer, antioxidant, antistress, and antiaging effects [11]. Previous studies have also reported that ginseng has antidepressant and antipsychotic effects. Ginseng extract and its ingredient ginsenosides increased the level of BDNF and its signaling pathway molecules and activated the neurogenesis in the hippocampus of depressive animals exposed to chronic mild stress $[12,13]$. In addition, ginseng decreased the schizophrenic symptoms in prenatally stressed neurodevelopmental and ketamine-treated schizophrenic rodents, regulating the neurodevelopmental genes and the levels of dopamine and 5-hydroxytryptamine $[14,15]$.

Based on various pharmacological properties of ginseng including beneficial effects on psychiatric diseases, in this 
study, we examined the effect of Panax ginseng Meyer (PG) extract on manic-like behavior in SD rats. In addition, in order to identify the molecular mechanism on the effect of PG on manic behaviors, we assessed differentially expressed genes (DEGs) by PG treatment in the prefrontal cortex of $\mathrm{SD}$ rats using RNA sequencing (RNA-seq). Among the DEGs, we particularly paid attention to the genes belonging to the glutamatergic synapse pathway, which showed the lowest $p$ value in the gene-enrichment analysis on DEGs based on the Kyoto Encyclopedia of Genes and Genomes (KEGG) pathways. We also focused on amphetamine addiction pathway genes because the amphetamine treatment in rodents has been widely used as a pharmacological mania model. The effect of PG on mania and BPD was further studied and discussed, focusing on the genes belonging to the glutamatergic synapse and amphetamine addiction pathways.

\section{Materials and Methods}

2.1. Preparation of PG. PG was purchased from Cheongsongyinsamsa (Kumsan, Chungnam, Korea) and was ground to powder. It $(300 \mathrm{~g})$ was extracted twice with $3 \mathrm{~L}$ of $70 \%$ ethyl alcohol for 1 day in a room temperature, filtered through filter paper (Advantec, Bunkyo-Ku, Tokyo, Japan), concentrated by rotary evaporator (N-1200BS, Eyela, Tokyo, Japan), and then freeze-dried (Freezone 6, Labconco, Kansas, MO, USA). The yield of freeze-dried PG was calculated to be $16.7 \%$.

2.2. Animals. Male Sprague-Dawley rats (150-200 g; Central Lab. Animal Inc., Seoul, Republic of Korea) were maintained under a 12-h light/dark cycle (light on at 7:00 AM and off at 7:00 PM) at a standard temperature $\left(25 \pm 2^{\circ} \mathrm{C}\right)$ with food and water freely available. All animal experiments were conducted in accordance with the animal care guidelines of the National Institute for Health (NIH) Guide and approved by the Animal Care and Use Committee at Semyum University (smecae17-07-02).

2.3. SD and PG Treatment. For REM-SD, the modified multiple platform method was used $[10,16]$. We randomly divided rats into control (non-SD), SD, and PG-treated SD groups. The rats of the SD and PG-treated SD groups were put in a chamber with six small platforms $(6 \mathrm{~cm}$ diameter $)$ surrounded by water ( $n=4$ per chamber). When the rats enter REM sleep, the rats were awakened when they touch the water by diminished muscle tone. The rats of the control group were put in a chamber with six large platforms $(15 \mathrm{~cm}$ diameter) ( $n=4$ per chamber). On large platforms, the rats could sleep on. But those are also surrounded by water and not enough for rats to walk around on. The water was filled up to about $2 \mathrm{~cm}$ below the surfaces of the platforms, and the rats could move across platforms within the chamber with large or small platforms. Food and water were available ad libitum. All rats remained in the chambers for $48 \mathrm{~h}$.

PG has been used as a treatment in CNS disease rats such as psychiatric and neurological diseases at doses of 100$200 \mathrm{mg} / \mathrm{kg}$ [17-19]. Based on these previous reports, we treated PG $(200 \mathrm{mg} / \mathrm{kg}$, in a concentration of $40 \mathrm{mg} / \mathrm{ml}$ in distilled water, p.o.) to rats of the PG-treated SD group, 5 times for a total period of 4 days, from 2 days prior to the start of SD (9:00 AM) to the end of SD at 9:00 AM. To the rats of the control and SD groups, distilled water was taken in the same manner. After $48 \mathrm{~h}$, the rats of all groups were sacrificed. The prefrontal cortex from each rat was dissected out, weighed, and kept frozen until analysis.

2.4. Elevated Plus Maze (EPM) Test. After SD for $48 \mathrm{~h}$, the EPM test was carried out in the manner of the previous study [20]. The EPM test apparatus was consisted of two open arms $(30 \times 10 \mathrm{~cm})$ and two enclosed arms $(30 \times 10 \times 30 \mathrm{~cm}$, with an open roof) of black wood and raised to a height of $50 \mathrm{~cm}$. After habituation in the testing room for $20 \mathrm{~min}$, the rats were put in the center of a cross-maze facing the open arms. For $5 \mathrm{~min}$ (test session), we checked the time spent and the numbers of entries into the open or closed arms.

2.5. RNA Isolation and RNA-Seq. The next-generation sequencing was performed using the RNA pooled from 3 rats per group. The total RNA was isolated from the prefrontal cortex samples pooled in each group using TRIzol. RNA integrity was measured using an Agilent 2100 Bioanalyzer (Agilent Technologies, Inc., Santa Clara, CA, USA).

The cDNA libraries were constructed using the TruSeq RNA Library kit on $1 \mu \mathrm{g}$ of total RNA according to the following process: extraction of polyA-selected RNA, fragmentation of RNA, reverse transcription using random hexamer, and 100 nt paired-end sequencing using Illumina HiSeq4000. We quantified the libraries through qPCR using an Agilent Technologies 2100 Bioanalyzer.

The low quality and adapter sequences were excluded from the raw reads of the sequencer. And then, the reads were aligned to the Rattus norvegicus (UCSC Baylor 3.4/rn4) using HISAT v2.0.5 [21]. The reference genome sequence of Rattus norvegicus and annotation data were acquired from the NCBI. The aligned reads were assembled into the transcripts, and their abundance was estimated using StringTie v1.3.3d [22, 23]. The relative abundance was estimated as fragments per kilobase of exon per million fragments mapped reads (FPKM) of transcript and gene expressed in each group. The RNAseq data has been uploaded to the public repository Sequence Read Archive (SRA) (https://submit.ncbi.nlm.nih.gov; submission ID: SUB8063770).

2.6. Statistical Analysis of Gene Expression Level. In order to select differentially expressed genes, the relative abundances for each gene were compared between groups. Genes whose FRKM was 0 in any of the groups were excluded from the analysis. After 1 was added to the FPKM values of genes, the values were converted based on $\log _{2}$ and then were subjected to quantile normalization. We determined the differential expression data by $\mid$ fold change (FC) $\mid \geq 2$ and independent $t$-test. In order to measure the similarity, hierarchical clustering for the DEG set was conducted using complete linkage and Euclidean distance. The gene-enrichment analysis for DEGs was also conducted based on the KEGG pathway (https://www.genome.jp/kegg/) database. Enrichment $p$ values on KEGG pathway terms were calculated 
based on a modified Fisher's exact test. And then, the false discovery rate (FDR-) adjusted $p$ values using the Benjamini-Hochberg algorithm were calculated. All data analysis on RNA-seq was conducted using R 3.4.3 (http:// www.r-project.org/).

2.7. Quantitative Real-Time PCR ( $q R T-P C R)$. cDNA was synthesized from total RNA using a 1st strand cDNA Synthesis Kit (BioAssay Co., Daejeon, Republic of Korea) according to the manufacturer's instructions. qRT-PCR was conducted using Real-Time PCR EvaGreen Kit (SolGent, Daejeon, Republic of Korea) and specific primers on each gene (Table S3). The analyses were performed using a StepOnePlus Real-Time PCR System (Applied Biosystems Inc., Carlsbad, CA, USA). The relative quantification of mRNA transcripts was calculated based on the $2^{-\Delta \Delta C T}$ method [24]. The expressions of UBC as a housekeeping gene were used for the analysis of relative expression levels of other genes [25].

2.8. Immunoblot. The prefrontal cortex dissected from each rat was homogenized in RIPA buffer with $1 \times$ protease/phosphatase inhibitor cocktail (Cell Signaling Technology, Beverly, MA, USA). For assessing the protein concentration, the Bradford reagent (Sigma-Aldrich, St. Louis, MO, USA) was used. After separated on sodium dodecyl sulfate (SDS-) polyacrylamide gels, proteins of $50 \mu \mathrm{g}$ were transferred onto a nitrocellulose membrane (Amersham Biosciences, Uppsala, Sweden). The membranes were incubated with mouse solute carrier family 1 (glial high-affinity glutamate transporter), member 2 (Slc1a2; also known as Eaat2) (Merck Millipore Darmstadt, Germany), rabbit Slc1a3 (also known as Eaat1), rabbit activity-regulated cytoskeleton-associated protein (Arc), rabbit $N$-methyl-D-aspartate (NMDA) receptor subunit 2A (NR2A), rabbit phospho-NR2A, rabbit NR2B (Y1325, Abcam, Cambridge, UK), and rabbit phosphoNR2B (Y1472; Cell Signaling Technology). Then, the membranes were further incubated with horseradish peroxidaseconjugated antimouse or antirabbit IgG (Santa Cruz Biotechnology, Santa Cruz, CA, USA). ECL substrate (Bio-Rad Laboratories, Hercules, CA, USA) was used for the visualization of protein bands. The band intensity was quantified using the ImageJ image analysis software (version 1.4; NIH).

2.9. Statistical Analysis. The results are expressed as mean \pm standard error of the mean (SE). The data were analyzed by one-way ANOVA followed by the LSD post hoc test, using the IBM SPSS Statistics 23 (SPSS Inc., Chicago, IL, USA). Values of $p<0.05$ were considered as statistically significant.

\section{Results}

3.1. Effect of PG on Manic-Like Behavior of SD Rats. The effect of PG on the hyperactive- and impulsive-like behaviors was assessed in SD rats using the EPM test. In the EPM test, the increased frequencies of open arm entries and the spent time in open arms have been considered as a sign of impulsive-like behavior [26, 27]. As shown in Figure 1, the SD rats entered the open arms more frequently and spent more time in the open arms than the control rats. In compar- ison, the PG-treated SD rats showed the decreased frequency of entries into and spent time within the open arms compared to the SD rats (Figures 1(a) and 1(b)). This result indicated that the PG treatment alleviated the increased impulsivity in SD rats. In addition, the PG treatment reduced the hyperlocomotion shown in SD rats. The frequency of the total entries to the open and close was increased in SD rats compared to control rats, whereas PG-treated SD rats revealed a lower frequency of total entries than SD rats (Figure 1(c)).

3.2. Altered Gene Expression by PG in the Prefrontal Cortex of $S D$ Rats. In order to identify the alteration of gene expressions by PG treatment in SD rats, we performed RNA-seq in the prefrontal cortex of the control, SD, and PG-treated SD rats. Through RNA-seq, we acquired the expression data on 13,254 genes. In the DEG analysis on RNA-seq result, we selected genes showing $|\mathrm{FC}|$ of 2.0 or greater and the a $p$ value of 0.05 or less in comparisons among groups. A total of 66 DEGs were identified in the control vs. SD comparison (Table S1). The expressions of 31 genes were upregulated in the $\mathrm{SD}$ rats compared to control rats, and the expressions of 35 genes were downregulated. In the SD vs. PG-treated SD comparison, 94 DEGs were identified (Table S2). PG treatment increased the expressions of 56 genes and decreased the expressions of 38 genes in SD rats. In order to determine the effect of PG on SD, we focused on 94 DEGs identified in the SD vs. PG-treated SD comparison and carried out a gene-enrichment analysis on 94 DEGs. Through the enrichment analysis based on the KEGG pathway database, 26 significant pathways were detected (FDR-corrected $p<0.05$; Table 1). Among the pathways, the glutamatergic synapse pathway showed the most significant enrichment. The glutamatergic synapse pathway genes altered by PG treatment in SD rats included Slcla2, Slc1a3, adenylate cyclase 1 (Adcy1), glutamate ionotropic receptor kainate type subunit 3 (Grik3), glutamate ionotropic receptor NMDA type subunit 3A (Grin3a), and G protein subunit gamma 2 (Gng2). In PG-treated SD rats, the expressions of all of these genes were elevated, compared to SD rats (Table 2). We also paid attention to the amphetamine addiction pathway (Table 1), because the amphetamine treatment in rodents has been widely used as a pharmacological mania model. Among the genes belonging to the amphetamine addiction pathway, the expressions of calcium/calmodulin-dependent protein kinase II alpha (Camk2a) and Grin3a were increased in PG-treated rats compared to SD rats, whereas the expression of Arc was decreased (Table 2). Among the genes belonging to the glutamatergic synapse or amphetamine addiction pathways, Slc1a3, Gng2, and Arc were also identified as DEGs in the control vs. SD comparison. In the SD rats, the expression of Slcla3 and Gng2 was downregulated, and the expression of Arc was upregulated, compared to control rats (Table 2).

3.3. Effect of $P G$ on the Expressions of the Glutamatergic Synapse and Amphetamine Addiction Pathway Genes in SD Rats. To validate the RNA-seq result, we examined the 


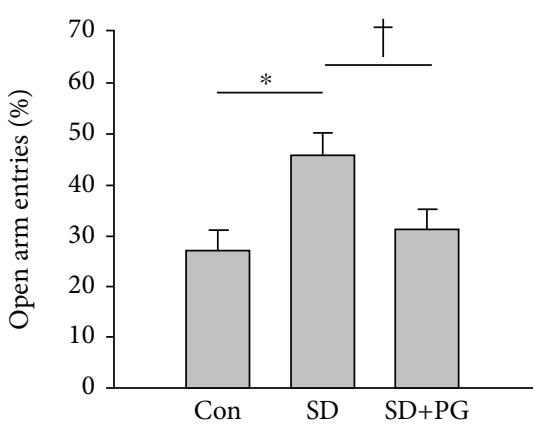

(a)

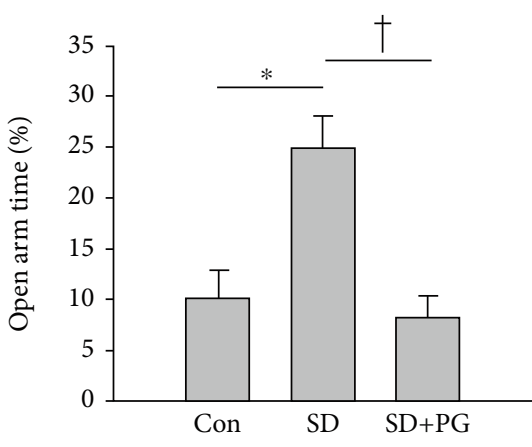

(b)

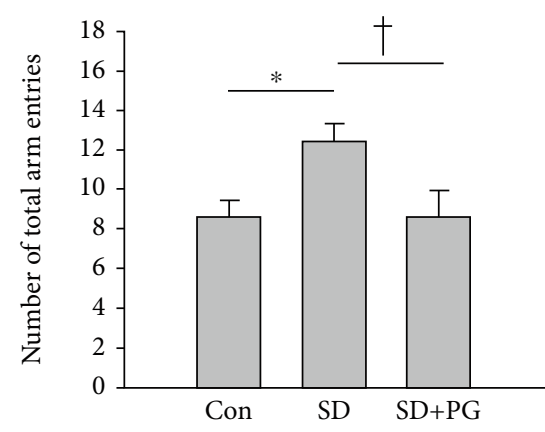

(c)

FIGURE 1: Effects of Panax ginseng (PG) on manic-like behaviors in sleep deprivation (SD) rats. Manic-like behaviors in sleep deprivation rats were measured using the elevated plus-maze (EPM) test. Impulsive behavior was indicated by (a) the percentage of open arm entries and (b) percentage of time spent in the open arms. (c) Hyperactive locomotion was indicated by the number of total entries into the open and closed arms. The results are presented as the mean \pm SE of two independent experiments $\left(n=4\right.$ for each group per each experiment). ${ }^{*} p<0.05$ compared to the control group; ${ }^{\dagger} p<0.05$ compared to the SD group. PG, PG.

TABle 1: Gene-enrichment analysis on differentially expressed genes (DEGs) by Panax ginseng (PG) in sleep deprivation (SD) rats.

\begin{tabular}{|c|c|c|c|c|c|}
\hline KEGG MapID & Map name & No. of genes & Genes & $p$ value & $\begin{array}{c}\text { FDR } \\
p \text { value }\end{array}$ \\
\hline 04724 & Glutamatergic synapse & 6 & $\begin{array}{l}\text { Grin3a, Slc1a2, Slc1a3, } \\
\text { Grik3, Adcy1, Gng2 }\end{array}$ & $<0.0001$ & 0.0001 \\
\hline 04371 & Apelin signaling pathway & 6 & Nos1, Spp1, Prkce, Adcy1, Klf2, Gng2 & $<0.0001$ & 0.0002 \\
\hline 05418 & Fluid shear stress and atherosclerosis & 5 & Dusp1, Mgst1, Bmp4, Klf2, Bmpr1b & 0.0001 & 0.0027 \\
\hline 04062 & Chemokine signaling pathway & 5 & Shc3, Ccl4, Adcy1, Gng2, Gsk3b & 0.0001 & 0.0037 \\
\hline 04024 & cAMP signaling pathway & 5 & Grin3a, Pde4d, Camk2a, Atp2b4, Adcy1 & 0.0001 & 0.0043 \\
\hline 05032 & Morphine addiction & 4 & Pde4d, Gabrb2, Adcy1, Gng2 & 0.0003 & 0.0072 \\
\hline 04713 & Circadian entrainment & 4 & Nos1, Camk2a, Adcy1, Gng2 & 0.0003 & 0.0073 \\
\hline 04919 & Thyroid hormone signaling pathway & 4 & Thrb, Bmp4, Pdpk1, Gsk3b & 0.0006 & 0.0105 \\
\hline 04722 & Neurotrophin signaling pathway & 4 & Shc3, Camk2a, Pdpk1, Gsk3b & 0.0007 & 0.0110 \\
\hline 04550 & $\begin{array}{l}\text { Signaling pathways regulating } \\
\text { pluripotency of stem cells }\end{array}$ & 4 & Bmp4, Inhba, Bmpr1b, Gsk3b & 0.0009 & 0.0135 \\
\hline 04261 & Adrenergic signaling in cardiomyocytes & 4 & Cacng4, Camk2a, Atp2b4, Adcy1 & 0.0010 & 0.0135 \\
\hline 04921 & Oxytocin signaling pathway & 4 & Cacng4, Camk2a, Adcy1, Myl6 & 0.0012 & 0.0146 \\
\hline 04390 & Hippo signaling pathway & 4 & Bmp4, Bmpr1b, Dlg2, Gsk3b & 0.0012 & 0.0146 \\
\hline 04360 & Axon guidance & 4 & Camk2a, Bmpr1b, Robo3, Gsk3b & 0.0018 & 0.0192 \\
\hline 05200 & Pathways in cancer & 5 & Bmp4, Adcy1, Zbtb16, Gng2, Gsk3b & 0.0019 & 0.0196 \\
\hline 04020 & Calcium signaling pathway & 4 & Nos1, Camk2a, Atp2b4, Adcy1 & 0.0021 & 0.0199 \\
\hline 04510 & Focal adhesion & 4 & Shc3, Spp1, Pdpk1, Gsk3b & 0.0026 & 0.0228 \\
\hline 05031 & Amphetamine addiction & 3 & Grin3a, Camk2a, Arc & 0.0037 & 0.0309 \\
\hline 04970 & Salivary secretion & 3 & Nos1, Atp2b4, Adcy1 & 0.0051 & 0.0402 \\
\hline 05206 & MicroRNAs in cancer & 4 & Mir27b, Mir128-1, Mir26a, Prkce & 0.0057 & 0.0419 \\
\hline 04925 & Aldosterone synthesis and secretion & 3 & Camk2a, Prkce, Adcy1 & 0.0058 & 0.0419 \\
\hline 04350 & TGF-beta signaling pathway & 3 & Bmp4, Inhba, Bmpr1b & 0.0062 & 0.0422 \\
\hline 04012 & ErbB signaling pathway & 3 & Shc3, Camk2a, Gsk3b & 0.0065 & 0.0422 \\
\hline 04727 & GABAergic synapse & 3 & Gabrb2, Adcy1, Gng2 & 0.0068 & 0.0422 \\
\hline 04080 & Neuroactive ligand-receptor interaction & 4 & Grin3a, Thrb, Gabrb2, Grik3 & 0.0070 & 0.0422 \\
\hline 04916 & Melanogenesis & 3 & Camk2a, Adcy1, Gsk3b & 0.0081 & 0.0470 \\
\hline
\end{tabular}

Gene-enrichment analysis for DEGs was performed based on the KEGG pathway database (https://www.genome.jp/kegg/). The $p$ values were calculated based on a modified Fisher's exact test. The false discovery rate (FDR) was controlled by adjusting the $p$ value using the Benjamini-Hochberg algorithm. 
TABLE 2: Different expressions of glutamatergic synapse and amphetamine addiction pathway genes in the control, sleep deprivation (SD), and Panax ginseng (PG-) treated SD rats.

\begin{tabular}{|c|c|c|c|}
\hline \multirow{2}{*}{ Gene } & \multirow{2}{*}{ Description } & \multicolumn{2}{|c|}{ FC } \\
\hline & & $\mathrm{SD}+\mathrm{PG} / \mathrm{S}$ & $\mathrm{SD} /$ control \\
\hline \multicolumn{4}{|c|}{ Glutamatergic synapse } \\
\hline Slcla3 & Solute carrier family 1 member 3 & 5.132 & -3.718 \\
\hline Slc1a2 & Solute carrier family 1 (glial high-affinity glutamate transporter), member 2 & 2.181 & -1.18 \\
\hline Adcy1 & Adenylate cyclase 1 (brain) & 3.268 & -1.125 \\
\hline Grik3 & Glutamate ionotropic receptor kainate type subunit 3 & 2.032 & 1.517 \\
\hline Grin3a & Glutamate ionotropic receptor NMDA type subunit $3 \mathrm{~A}$ & 2.012 & -1.283 \\
\hline Gng2 & G protein subunit gamma 2 & 62.921 & -53.461 \\
\hline \multicolumn{4}{|c|}{ Amphetamine addiction } \\
\hline Camk2a & Calcium/calmodulin-dependent protein kinase II alpha & 2.111 & -1.135 \\
\hline Arc & Activity-regulated cytoskeleton-associated protein & -2.096 & 3.674 \\
\hline Grin3a & Glutamate ionotropic receptor NMDA type subunit $3 \mathrm{~A}$ & 2.012 & -1.283 \\
\hline
\end{tabular}

FC: fold-change.
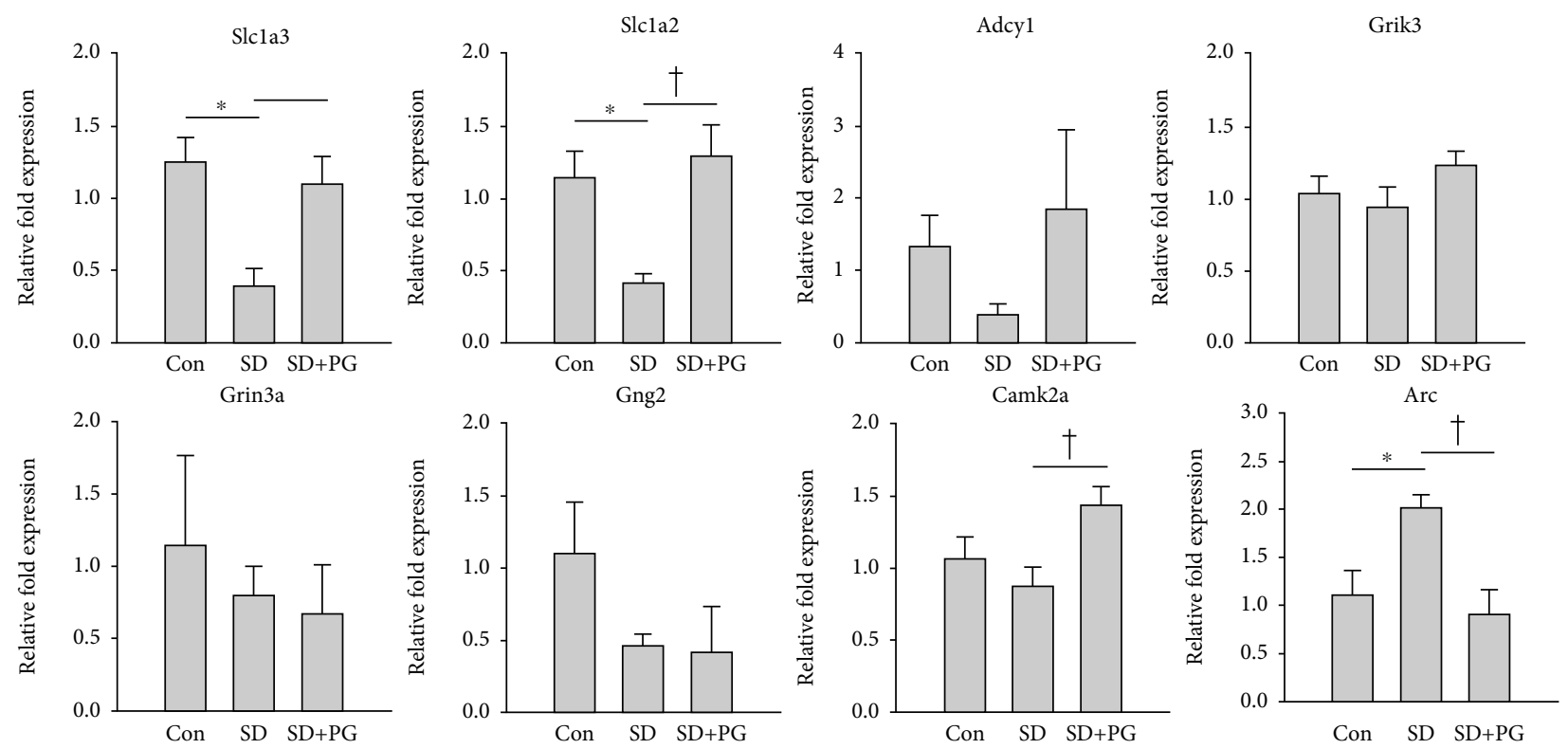

FIGURE 2: Quantitative real-time PCR (qRT-PCR) validation of the glutamatergic synapse and amphetamine addiction pathway genes. Expressions of the glutamatergic synapse and amphetamine addiction pathway genes were determined using the qRT-PCR in the prefrontal cortex of the control, sleep deprivation (SD), and Panax ginseng (PG)-treated SD rats. The histogram reveals the expression levels of genes as mean \pm SE. Expression levels were normalized against ubiquitin $\mathrm{C}$ (Ubc). The experiments were repeated in duplicates ( $n=4$ for each group per experiment). ${ }^{*} p<0.05$ compared to the control group; ${ }^{\dagger} p<0.05$ compared to the SD group.

expressions of 8 genes belonging to the glutamatergic synapse and amphetamine addiction pathways in the prefrontal cortex of the control, SD, and PG-treated SD rats using qRT-PCR. As shown in Figure 2, the mRNA expressions of Slc1a3 and Slcla2 were decreased in SD rats compared to the control rats, and the PG treatment alleviated the decreases. In contrast, SD elevated the expression of Arc, and the PG treatment inhibited the elevation of Arc in SD rats. The expression level of Camk2a was higher in the PG-treated SD rats than in SD rats, but the difference of the expressions between the control and SD rats was not observed. Although our RNA-seq result showed that the expressions of Adcy1, Grik3, Grin3a, and Gng2 were upregulated in PG-treated SD rats compared to SD rats, the significant difference of their expressions was not detected in the qRT-PCR analysis.

3.4. Effect of PG on the Protein Expressions of Slc1a2, Slc1a3, and Arc in SD Rats. We also examined the protein expressions of Slc1a2, Slc1a3, and Arc in the prefrontal cortex of the control, SD, and PG-treated SD rats (Figures 3(a) and 3(b)). The protein expressions of Slc1a2 and Slcla3 were reduced in SD rats, whereas the PG treatment restored the reduction in SD rats. In contrast, SD enhanced the protein 


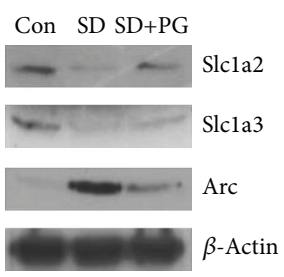

(a)

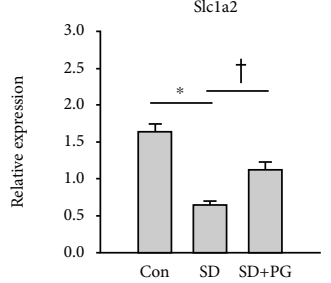

Con $\quad$ SD $\quad$ SD+PG

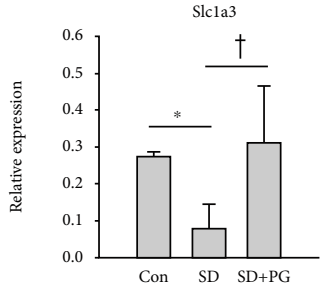

(b)
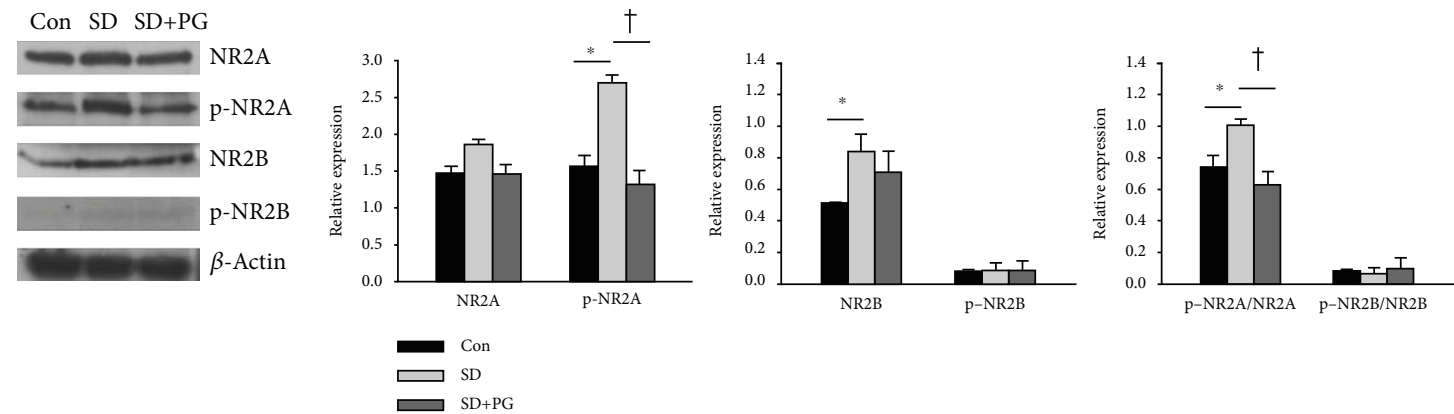

(d)

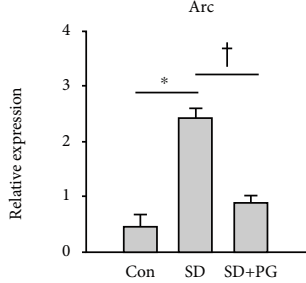

(c)

Figure 3: Effect of Panax ginseng (PG) on the expression of Slc1a2, Slc1A3, and Arc and the phosphorylation of the NMDA receptor in sleep deprivation (SD) rats. (a) The expressions of Slc1a2, Slc1A3, and Arc and (b) the phosphorylations of the NR2 subunits of the NMDA receptor were determined using immunoblotting in the prefrontal cortex of the control, SD, and PG-treated SD rats. The left and middle histograms represent the protein expression and the phosphorylation levels divided by the expression level of $\beta$-actin as mean \pm SE. The right histogram shows the ratio of the phosphorylation levels to the protein expression levels. $\beta$-Actin expression was used as an internal control. The experiments were repeated in duplicates $\left(n=3\right.$ for each group per experiment). ${ }^{*} p<0.05$ compared to the control group; ${ }^{\dagger} p<0.05$ compared to the SD group.

expression of Arc, whereas the PG treatment in SD rats suppressed the enhanced expression of Arc.

3.5. Effect of PG on the Activation of NMDA Receptors in SD Rats. In order to determine the effect of PG on the activation of NMDA receptor, which is a key receptor in neurotransmission through the glutamatergic synapse and amphetamine addiction pathways, we assessed the expression and phosphorylation levels of NR2A and NR2B subunits of the NMDA receptor in the prefrontal cortex of the control, SD, and PG-treated SD rats. As shown in Figures 3(c) and 3(d), $\mathrm{SD}$ and $\mathrm{PG}$ treatment did not affect the protein expression of NR2A. However, SD could elevate the phosphorylation of NR2A, whereas PG inhibited the SD-induced phosphorylation of NR2A. In addition, SD increased the protein expression of NR2B. However, the PG treatment did not suppress the expression of NR2B. Neither SD nor PG treatment affects the phosphorylation of NR2B. These results indicate that the PG treatment could inhibit the SD-induced activation of the NR2A subunit of the NMDA receptor in the prefrontal cortex, but not the NR2B subunit.

\section{Discussion}

In this study, we found that PG could attenuate the maniclike behaviors shown in SD rats. In order to identify the mechanism of the effect of PG in SD rats, we assessed the alteration of molecules by PG treatment in the prefrontal cortex of SD rats. PG prominently altered the expressions of the glutamatergic synapse and amphetamine addiction pathway genes in SD rats. In particular, PG alleviated the decreased expressions of Slcla2 and Slcla3 and the increased expression of Arc shown in SD rats. These alleviations by PG in $\mathrm{SD}$ rats were also observed in the protein levels. Moreover, PG could inhibit the SD-induced phosphorylation of the NR2A subunit of the NMDA receptor.

BPD patients in a manic state represent the characteristic behaviors such as hyperactivity, impulsivity, and aggressiveness $[28,29]$. Rodent models of mania have also shown the hyperactive- and the impulsive-like behaviors, increasing the total number of entries into the arms and the frequencies of the open arm entries/the spent time in the open arms in the EPM test [30-32]. Also, in our SD rats, the hyperactive and impulsive mania-like behaviors were observed through the EPM test, similar to the mania rodent models of previous studies. Interestingly, PG treatment significantly reduced these mania-like behaviors. A lot of studies have reported the effect of ginseng and ginsenosides on the hyperactive/impulsive behaviors in psychiatric diseases and conditions. Clinical studies showed that PG and Korean red ginseng (KRG) alleviated the hyperactivity/impulsivity in patients with attention deficit hyperactivity disorder (ADHD) [33, 34]. Additionally, in animal studies, ginsenosides relieved the hyperactive behaviors, along with reducing the behavioral sensitization and conditioned place preference in cocaine- [35], methamphetamine- [36], morphine- [37], and nicotine-treated mice [38, 39]. In addition to these behavioral effects, several studies reported that ginseng and ginsenosides could suppress the enhanced central dopaminergic transmission including dopamine-releasing and/or 
binding to dopamine receptors [36-39]. However, the mechanism on the alleviating effect of ginseng or ginsenosides against manic behaviors is still not largely elucidated.

In our study, through RNA-seq, we found that PG could regulate potently the glutamatergic synapse pathway genes in the prefrontal cortex of SD rats. The expressions of Slcla3, Slc1a2, Adcy1, Grik3, Grin3a, and Gng2 were increased by the PG treatment in SD rats. We also paid attention to the amphetamine addiction pathway genes such as Camk2a, Arc, and Grin3a. The expressions of Camk2a and Grin3a were upregulated by the PG treatment in SD rats, whereas the expression of Arc was downregulated. Among these genes, we expected that Slcla3, Gng2, and Arc might be candidate genes related to the alleviating effect of PG on maniclike behaviors of SD rats. Intriguingly, these genes were not only detected as DEGs in the comparison between SD and control but also PG treatment restored the expression changes of these genes in SD rats. In qRT-PCR, we also observed the expression changes on Slc1a3 and Arc, similar to the RNA-seq result, but not on Gng2. SD attenuated the expression of Slc1a3 and enhanced the expression of Arc, whereas PG relieved SD-induced expression changes. Moreover, we found the additional candidate gene Slc1a2. Although in the DEG analysis between the control and SD, Slc1a2 was excluded by $|\mathrm{FC}|$ cutoff of $\geq 2 \quad(\mathrm{FC}=-1.7$; Table 2); qRT-PCR showed that the expression of Slcla2 was significantly reduced by $\mathrm{SD}$ and PG ameliorated the reduced expression, consistent with our RNA-seq result. However, differently from the RNA-seq result, the expressions of Adcy1, Grik3, Grin3a, and Gng2 were not upregulated by PG treatment in SD rats. This inconsistency may be attributed to the expression volume of the gene that was the geometric mean of the two groups' expression levels. Even though $|\mathrm{FC}|$ might be different by twofold, the expression change of gene with higher volume may be more credible [40]. In our RNA-seq result, Slc1a3, Slc1a2, Camk2a, and Arc showed higher expression volumes than Adcy1, Grik3, Ginr3a, and Gng2 (data not shown). Although the expression changes of Adcyl, Grik3, Grin3a, and Gng2 were not detected in qRT-PCR (Figure 2); instead, we could acquire the confidence on the expression changes of Slcla3, Slc1a2, Camk2a, and Arc. We focused on Slc1a3, Slc1a2, and Arc and examined their protein expressions. SD reduced the protein expressions of Slc1a3 and Slcla2 and elevated the expression of Arc. In comparison, PG treatment inhibited SDinduced these expression changes (Figures $3(\mathrm{a})$ and $3(\mathrm{~b})$ ).

The concentrations of glutamate in the extracellular space are maintained by glutamate transporters. Glutamate transporters, Slcla and Slcla3, were potently located in the plasma membranes of astrocytes and oligodendrocytes. Those remove approximately $95 \%$ of the extracellular glutamate [41]. The glutamatergic neurotransmission has been involved in the pathophysiology and treatment of mania and BPD. The elevated levels of glutamate have been reported in patients with BPD [42, 43]. A mood stabilizer, valproic acid, was reported to increase the levels of Slcla2 and Slc1a3 and the capacity of glutamate uptake in the rat hippocampus [44] and also to decrease the glutamate levels in mice whole brain [45]. Given these reports, PG may atten- uate the synaptic glutamate level, enhancing the glutamate uptake via increasing the expression levels of Slcla and Slc1a3 in SD rats. Thus, it may contribute to suppressing the glutamatergic synaptic activity, resulting in decreasing the mRNA and protein levels of Arc in SD rats.

The immediate-early gene Arc, which is considered as a plasticity-related gene, plays a key role in the long-term potentiation, long-term depression, and synaptic transmission $[46,47]$. Glutamate could increase the mRNA and protein expression of Arc in neurons. The increase of Arc expression in the excitatory neurons was dependent on NMDA receptor, which is one of three types of ionotropic glutamate receptors $[48,49]$. In comparison, another ionotropic glutamate receptor, alpha-amino-3-hydroxy-5-methyl-4isoxazolepropionic acid (AMPA) receptor, was reported to negatively regulate the Arc transcription or not to affect the level of Arc $[48,50]$. Given these previous reports, the alteration of Arc expressions in SD and PG-treated SD rats may be mediated via the regulation of NMDA receptor activity.

The NMDA receptor forms a tetramer by three different subunits: NR1, NR2, and NR3. Heterotetrameric NMDA receptor is broadly expressed in the brain, which consisted of two obligatory glycine-binding NR1 subunits and two regionally localized glutamate-binding NR2 subunits, particularly NR2A and NR2B. We examined the effect of PG on the NMDA receptor, focusing on the NR2 subunits containing the glutamate binding site. Previous studies have reported the involvement of the phosphorylation of NR2 subunits in the action of lithium. Lithium inhibited the tyrosine phosphorylation of the NR2A subunit, together with the decreased interaction of NR2A with the Src family tyrosine kinases in the rat hippocampus following cerebral ischemia [51]. Lithium also suppressed the tyrosine phosphorylation of the NR2B subunit in cultured cortical neurons [52]. The tyrosine phosphorylations of NR2A including Y1325 could potentiate the NMDA receptor currents $[53,54]$. In comparison, the phosphorylation of NR2B Y1472 inhibited the endocytosis of the NR2B-containing NMDA receptor, leading to the stabilization of the receptor on the cell surface [55]. Interestingly, we found that SD increased the tyrosine phosphorylation of the NR2A subunit (Y1325), whereas PG alleviated the SD-induced phosphorylation. However, the phosphorylation of the NR2B subunit (Y1472) was induced by neither SD nor PG treatment. Therefore, our result indicated that PG might relieve the glutamate neurotransmission activated in SD rats, regulating the potential of the NMDA receptor currents rather than the surface expression or stabilizing of the receptor. There were some limitations in our RNAseq result. In the DEG analysis, we did not determine the differential expressions by FDR-corrected $p$ value $<0.05$. However, after selecting the candidate genes related to the effect of PG on SD of DEGs, we validated the mRNA expressions of candidate genes using the qRT-PCR as well as the expression levels of proteins encoded by the genes using immunoblotting.

\section{Conclusions}

In the present study, we found that PG could increase the mRNA and protein expressions of Slc1a3 and Slc1a2 in the 
prefrontal cortex of PG-treated SD rats. The increase of the expressions of Slc1a3 and Slcla2 by PG may lead to the decrease of the extracellular glutamate levels. In turn, it would reduce the NMDA receptor activation and the expression level of Arc in SD rats. The attenuated glutamatergic neurotransmission by PG would contribute to the reduction of mania-like behaviors in SD rats. These results suggested the useful therapeutic effect of PG on the management of a manic phase of BPD.

\section{Data Availability}

The data used to support the findings of this study are available from the corresponding author upon request.

\section{Conflicts of Interest}

The authors declare no conflict of interest.

\section{Authors' Contributions}

HJP designed and supervised this study. KHL, SAK, and HJP conducted the experimental work and analyzed data. KHL drafted the manuscript. All authors contributed to the writing of the manuscript and approved the final manuscript.

\section{Acknowledgments}

This work was supported by the National Research Foundation of Korea (NRF) grant funded by the Korea government (2017R1C1B2005802).

\section{Supplementary Materials}

Supplemental Table S1. Differentially expressed genes between control and sleep deprivation (SD) rats. Supplemental Table S2. Differentially expressed genes between sleep deprivation (SD) and Panax ginseng (PG)-treated SD rats. Supplemental Table S3. Primer sequences used for qRTPCR. (Supplementary Materials)

\section{References}

[1] L. G. Sylvia, J. M. Dupuy, M. J. Ostacher et al., "Sleep disturbance in euthymic bipolar patients," Journal of Psychopharmacology, vol. 26, no. 8, pp. 1108-1112, 2011.

[2] K. A. Kaplan, M. G. EL, A. Soehner et al., "Hypersomnia subtypes, sleep and relapse in bipolar disorder," Psychological Medicine, vol. 45, no. 8, pp. 1751-1763, 2015.

[3] R. W. Logan and C. A. McClung, "Animal models of bipolar mania: the past, present and future," Neuroscience, vol. 321, pp. 163-188, 2016.

[4] J. W. Young, B. L. Henry, and M. A. Geyer, "Predictive animal models of mania: hits, misses and future directions," British Journal of Pharmacology, vol. 164, no. 4, pp. 1263-1284, 2011.

[5] E. Abrial, A. Bétourné, A. Etiévant et al., "Protein kinase C inhibition rescues manic-like behaviors and hippocampal cell proliferation deficits in the sleep deprivation model of mania," International Journal of Neuropsychopharmacology, vol. 18, no. 2, 2014 .
[6] E. L. Streck, G. Scaini, G. C. Jeremias et al., "Effects of mood stabilizers on brain energy metabolism in mice submitted to an animal model of mania induced by paradoxical sleep deprivation," Neurochemical Research, vol. 40, no. 6, pp. 1144-1152, 2015.

[7] F. Armani, M. L. Andersen, R. Andreatini, R. Frussa-Filho, S. Tufik, and J. C. Fernandes Galduróz, "Successful combined therapy with tamoxifen and lithium in a paradoxical sleep deprivation-induced mania model," CNS Neuroscience \& Therapeutics, vol. 18, no. 2, pp. 119-125, 2012.

[8] Y. J. Chiou and T. L. Huang, "Brain-derived neurotrophic factor (BDNF) and bipolar disorder," Psychiatry Research, vol. 274, pp. 395-399, 2019.

[9] S. A. Siddique, T. Tamilselvan, M. Vishnupriya, and E. Balamurugan, "Evaluation of neurotransmitter alterations in four distinct brain regions after rapid eye movement sleep deprivation (REMSD) induced mania-like behaviour in Swiss albino mice," Neurochemical Research, vol. 43, no. 6, pp. 1171-1181, 2018.

[10] G. L. Gessa, L. Pani, P. Fadda, and W. Fratta, "Sleep deprivation in the rat: an animal model of mania," European Neuropsychopharmacology, vol. 5, Supplement, pp. 89-93, 1995.

[11] A. S. Attele, J. A. Wu, and C. S. Yuan, "Ginseng pharmacology: multiple constituents and multiple actions," Biochemical Pharmacology, vol. 58, no. 11, pp. 1685-1693, 1999.

[12] D. Xu, C. Wang, W. Zhao, S. Gao, and Z. Cui, "Antidepressant-like effects of ginsenoside Rg5 in mice: involving of hippocampus BDNF signaling pathway," Neuroscience Letters, vol. 645, pp. 97-105, 2017.

[13] B. Jiang, Z. Xiong, J. Yang et al., "Antidepressant-like effects of ginsenoside Rg1 are due to activation of the BDNF signalling pathway and neurogenesis in the hippocampus," British Journal of Pharmacology, vol. 166, no. 6, pp. 18721887, 2012.

[14] Y. O. Kim, H. Y. Lee, H. Won et al., "Influence of Panax ginseng on the offspring of adult rats exposed to prenatal stress," International Journal of Molecular Medicine, vol. 35, no. 1, pp. 103-109, 2015.

[15] M. Chatterjee, S. Singh, R. Kumari, A. K. Verma, and G. Palit, "Evaluation of the antipsychotic potential of Panax quinquefolium in ketamine induced experimental psychosis model in mice," Neurochemical Research, vol. 37, no. 4, pp. 759-770, 2012.

[16] K. Shahveisi, H. Khazaie, V. Farnia, and M. Khodamoradi, "REM sleep deprivation impairs retrieval, but not reconsolidation, of methamphetamine reward memory in male rats," Pharmacology Biochemistry and Behavior, vol. 185, article 172759, 2019.

[17] D. Jang and H. J. Lee, "White ginseng ameliorates depressive behavior and increases hippocampal 5-HT level in the stressed ovariectomized rats," BioMed Research International, vol. 2019, Article ID 5705232, 6 pages, 2019.

[18] C. R. Sanghavi, S. A. Barhate, M. S. Mahajan, M. Mohan, and S. B. Kasture, "Korean ginseng extract attenuates reserpineinduced orofacial dyskinesia and improves cognitive dysfunction in rats," Natural Product Research, vol. 25, no. 7, pp. 704715, 2011.

[19] A. Kumar, P. Rinwa, and H. Dhar, "Microglial inhibitory effect of ginseng ameliorates cognitive deficits and neuroinflammation following traumatic head injury in rats," Inflammopharmacology, vol. 22, no. 3, pp. 155-167, 2014. 
[20] H. J. Park, S. K. Kim, W. S. Kang, J. H. Chung, and J. W. Kim, "Increased activation of synapsin 1 and mitogen-activated protein kinases/extracellular signal-regulated kinase in the amygdala of maternal separation rats," CNS Neuroscience \& Therapeutics, vol. 20, no. 2, pp. 172-181, 2014.

[21] D. Kim, B. Langmead, and S. L. Salzberg, "HISAT: a fast spliced aligner with low memory requirements," Nature methods, vol. 12, no. 4, pp. 357-360, 2015.

[22] M. Pertea, G. M. Pertea, C. M. Antonescu, T. C. Chang, J. T. Mendell, and S. L. Salzberg, "StringTie enables improved reconstruction of a transcriptome from RNA-seq reads," Nature Biotechnology, vol. 33, no. 3, pp. 290-295, 2015.

[23] M. Pertea, D. Kim, G. M. Pertea, J. T. Leek, and S. L. Salzberg, "Transcript-level expression analysis of RNA-seq experiments with HISAT, StringTie and Ballgown," Nature Protocols, vol. 11, no. 9, pp. 1650-1667, 2016.

[24] K. J. Livak and T. D. Schmittgen, "Analysis of relative gene expression data using real-time quantitative PCR and the 2(Delta Delta C(T)) method," Methods, vol. 25, no. 4, pp. 402408, 2001.

[25] J. Vandesompele, K. De Preter, F. Pattyn et al., “Accurate normalization of real-time quantitative RT-PCR data by geometric averaging of multiple internal control genes," Genome biology, vol. 3, no. 7, article Research0034, 2002.

[26] A. F. Logsdon, B. P. Lucke-Wold, L. Nguyen et al., "Salubrinal reduces oxidative stress, neuroinflammation and impulsivelike behavior in a rodent model of traumatic brain injury," Brain Research, vol. 1643, pp. 140-151, 2016.

[27] V. Mosienko, B. Bert, D. Beis et al., "Exaggerated aggression and decreased anxiety in mice deficient in brain serotonin," Translational psychiatry, vol. 2, no. 5, article e122, 2012.

[28] P. Azzam, S. Obeid, C. Haddad et al., "Relationship between impulsivity and clinical and sociodemographic variables among Lebanese patients with bipolar disorder: results of a cross-sectional study," The Journal of Nervous and Mental Disease, vol. 207, no. 7, pp. 596-603, 2019.

[29] B. M. Stiles, A. F. Fish, and R. Vandermause, "Identifying the complexity of diagnosing bipolar disorder: a focused ethnography," Issues in Mental Health Nursing, vol. 40, no. 9, pp. 812818, 2019.

[30] S. Acharjee, W. G. Branton, P. Vivithanaporn et al., "HIV-1 Nef expression in microglia disrupts dopaminergic and immune functions with associated mania-like behaviors," Brain, Behavior, and Immunity, vol. 40, pp. 74-84, 2014.

[31] G. S. Kirshenbaum, C. R. Burgess, N. Dery, M. Fahnestock, J. H. Peever, and J. C. Roder, "Attenuation of mania-like behavior in $\mathrm{Na}+\mathrm{K}+$-ATPase $\alpha 3$ mutant mice by prospective therapies for bipolar disorder: melatonin and exercise," Neuroscience, vol. 260, pp. 195-204, 2014.

[32] T. Isozaki, S. Komenoi, Q. Lu et al., "Deficiency of diacylglycerol kinase $\eta$ induces lithium-sensitive mania-like behavior," Journal of Neurochemistry, vol. 138, no. 3, pp. 448-456, 2016.

[33] H. Niederhofer, "Panax ginseng may improve some symptoms of attention-deficit hyperactivity disorder," Journal of dietary supplements, vol. 6, no. 1, pp. 22-27, 2009.

[34] H. J. Ko, I. Kim, J. B. Kim et al., "Effects of Korean red ginseng extract on behavior in children with symptoms of inattention and hyperactivity/impulsivity: a double-blind randomized placebo-controlled trial," Journal of Child and Adolescent Psychopharmacology, vol. 24, no. 9, pp. 501-508, 2014.
[35] H. S. Kim, K. S. Kim, and K. W. Oh, "Inhibition by ginsenosides Rb1 and Rg1 of cocaine-induced hyperactivity, conditioned place preference, and postsynaptic dopamine receptor supersensitivity in mice," Pharmacology, Biochemistry, and Behavior, vol. 63, no. 3, pp. 407-412, 1999.

[36] H. S. Kim, C. G. Jang, W. K. Park et al., "Blockade by ginseng total saponin of methamphetamine-induced hyperactivity and conditioned place preference in mice," General Pharmacology, vol. 27, no. 2, pp. 199-204, 1996.

[37] H. S. Kim, C. G. Jang, K. W. Oh et al., "Effects of ginseng total saponin on morphine-induced hyperactivity and conditioned place preference in mice," Journal of Ethnopharmacology, vol. 60, no. 1, pp. 33-42, 1998.

[38] S. E. Kim, I. Shim, J. K. Chung, and M. C. Lee, "Effect of ginseng saponins on enhanced dopaminergic transmission and locomotor hyperactivity induced by nicotine," Neuropsychopharmacology, vol. 31, no. 8, pp. 1714-1721, 2006.

[39] H. S. Kim and K. S. Kim, "Inhibitory effects of ginseng total saponin on nicotine-induced hyperactivity, reverse tolerance and dopamine receptor supersensitivity," Behavioural Brain Research, vol. 103, no. 1, pp. 55-61, 1999.

[40] H. J. Park, J. M. Hong, J. H. Lee et al., "Comparative transcriptome analysis of skeletal muscle in ADSSL1 myopathy," Neuromuscular disorders, vol. 29, no. 4, pp. 274-281, 2019.

[41] J. D. Rothstein, M. Dykes-Hoberg, C. A. Pardo et al., "Knockout of glutamate transporters reveals a major role for astroglial transport in excitotoxicity and clearance of glutamate," Neuron, vol. 16, no. 3, pp. 675-686, 1996.

[42] S. R. Dager, S. D. Friedman, A. Parow et al., "Brain metabolic alterations in medication-free patients with bipolar disorder," Archives of General Psychiatry, vol. 61, no. 5, pp. 450-458, 2004.

[43] C. A. Altamura, M. C. Mauri, A. Ferrara, A. R. Moro, G. D'Andrea, and F. Zamberlan, "Plasma and platelet excitatory amino acids in psychiatric disorders," The American Journal of Psychiatry, vol. 150, no. 11, pp. 1731-1733, 1993.

[44] B. Hassel, E. G. Iversen, L. Gjerstad, and E. Taubøll, "Up-regulation of hippocampal glutamate transport during chronic treatment with sodium valproate," Journal of Neurochemistry, vol. 77, no. 5, pp. 1285-1292, 2001.

[45] C. U. Johannessen, D. Petersen, F. Fonnum, and B. Hassel, "The acute effect of valproate on cerebral energy metabolism in mice," Epilepsy Research, vol. 47, no. 3, pp. 247-256, 2001.

[46] C. R. Bramham, P. F. Worley, M. J. Moore, and J. F. Guzowski, "The immediate early gene arc/arg3.1: regulation, mechanisms, and function," The Journal of Neuroscience, vol. 28, no. 46, pp. 11760-11767, 2008.

[47] A. V. Tzingounis and R. A. Nicoll, "Arc/Arg3.1: linking gene expression to synaptic plasticity and memory," Neuron, vol. 52, no. 3, pp. 403-407, 2006.

[48] T. Chen, J. Zhu, L. K. Yang, Y. Feng, W. Lin, and Y. H. Wang, "Glutamate-induced rapid induction of Arc/Arg3.1 requires NMDA receptor-mediated phosphorylation of ERK and CREB," Neuroscience Letters, vol. 661, pp. 23-28, 2017.

[49] O. Steward and P. F. Worley, "Selective targeting of newly synthesized Arc mRNA to active synapses requires NMDA receptor activation," Neuron, vol. 30, no. 1, pp. 227-240, 2001.

[50] V. R. Rao, S. A. Pintchovski, J. Chin, C. L. Peebles, S. Mitra, and S. Finkbeiner, "AMPA receptors regulate transcription of the plasticity-related immediate-early gene Arc," Nature Neuroscience, vol. 9, no. 7, pp. 887-895, 2006. 
[51] J. Ma and G. Y. Zhang, "Lithium reduced N-methyl-D-aspartate receptor subunit $2 \mathrm{~A}$ tyrosine phosphorylation and its interactions with Src and Fyn mediated by PSD-95 in rat hippocampus following cerebral ischemia," Neuroscience Letters, vol. 348, no. 3, pp. 185-189, 2003.

[52] R. Hashimoto, C. Hough, T. Nakazawa, T. Yamamoto, and D. M. Chuang, "Lithium protection against glutamate excitotoxicity in rat cerebral cortical neurons: involvement of NMDA receptor inhibition possibly by decreasing NR2B tyrosine phosphorylation," Journal of Neurochemistry, vol. 80, no. 4, pp. 589-597, 2002.

[53] G. Kohr and P. H. Seeburg, "Subtype-specific regulation of recombinant NMDA receptor-channels by protein tyrosine kinases of the src family," The Journal of Physiology, vol. 492, no. 2, pp. 445-452, 1996.

[54] F. Zheng, M. B. Gingrich, S. F. Traynelis, and P. J. Conn, "Tyrosine kinase potentiates NMDA receptor currents by reducing tonic zinc inhibition," Nature Neuroscience, vol. 1, no. 3, pp. 185-191, 1998.

[55] E. M. Snyder, Y. Nong, C. G. Almeida et al., "Regulation of NMDA receptor trafficking by amyloid-beta," Nature Neuroscience, vol. 8, no. 8, pp. 1051-1058, 2005. 